Case Report

\title{
Giant cell tumor of the tenosynovial type in the prevertebral space with a cervical approach
}

\author{
Daniel A. Rappoport ${ }^{1}$, Lucas Fuenzalida ${ }^{2 *}$, Patricio I. Sepulveda ${ }^{3}$, Patricio J. Gac ${ }^{1}$ \\ ${ }^{1}$ Department of Surgery, ${ }^{2}$ Faculty of Medicine, ${ }^{1-3}$ Hospital Clínico Universidad de Chile, Santiago, Chile \\ Received: 11 November 2021 \\ Revised: 10 December 2021 \\ Accepted: 15 December 2021

\section{*Correspondence:} \\ Dr. Lucas Fuenzalida, \\ E-mail: lucas.fuenzalida@ug.uchile.cl \\ Copyright: () the author(s), publisher and licensee Medip Academy. This is an open-access article distributed under \\ the terms of the Creative Commons Attribution Non-Commercial License, which permits unrestricted non-commercial \\ use, distribution, and reproduction in any medium, provided the original work is properly cited.
}

\begin{abstract}
The complex anatomy of the neck makes surgical interventions of cervical neoplasms a very complex process. The aforementioned challenges the surgeon to find a surgical approach with the least morbidity and cosmetic sequelae. This is particularly true in tumoral occupation of the prevertebral space (PVS), most frequently due to the extension of neoplasms from naso and oropharynx; whereas primary tumors of the PVS are extremely rare. There are a number of surgical approaches to access the skull base and the anterior region of the upper cervical spine, such as the transcervicaltranstemporal approach, retraction and dissection of the soft palate, mandibular osteotomy, medial glossotomy, among others. The following article presents a case report in which a less invasive combined transcervical-transoral approach was performed on a tenosynovial giant cell tumor of the PVS. As an alternative in the treatment of these type of lesions, it is in our opinion equally effective and less morbid.
\end{abstract}

Keywords: Prevertebral space, Retropharyngeal space, Giant cell tumor, Neck masses, Dysphonia, Head and neck surgery

\section{INTRODUCTION}

The complex anatomy of the neck makes surgical interventions of cervical neoplasms and the whole diagnostic process very complex; specially when located in the retropharyngeal space (RPS) (or retro visceral space), danger space (DS), and the prevertebral space (PVS). The difficult access to this anatomical region challenges the surgeon to find a surgical approach with the least possible morbidity and cosmetic sequelae, a balance that is not easily obtained.

Our objective is to present a case in which a less invasive combined transcervical-transoral approach was performed, which in our opinion, is equally effective and less morbid, as an alternative in the treatment of this type of lesions.

\section{CASE REPORT}

A 44-year-old woman consults at the outpatient clinic with a history of a thyroid nodule with a Bethesda IV (fine needle aspiration), for which surgery was indicated. After questioning, the only symptom she reported was intermittent dysphonia. Nasofibrolaryngoscopy revealed a fibrovascular mass in the posterior pharyngeal wall, with asymmetric glottic closure that could be interpreted as a compensated left vocal cord paresis and chronic laryngopathy due to moderate pharyngolaryngeal reflux.

Computed tomography (CT) showed a lobulated mass located in the PVS at the height of $\mathrm{C} 2$, closely related to the insertion of the superior fascicles of longus colli muscle, causing osseous remodeling in the anterior face of the body of $\mathrm{C} 2$ and in the anterior tubercle of $\mathrm{C} 1$, without bone destruction or invasion of adjacent tissues (Figure 1). In addition, the adipose tissue of the RPS was displaced 
anteriorly and the caliber of the airway decreased without lymph node metastases. Magnetic resonance (MR), showed an heterogeneous and hypointense signal in T2weighted sequences of the mass, suggesting a fibrous component which confirmed the location and nonaggressive appearance of the mass, closely related to the myo-tendon insertion of the longus colli muscle, without evidence of bone invasion (Figure 2). Due to its imaging characteristics, differential diagnosis included extra-articular pigmented villonodular synovitis and tenosynovial giant cells tumor.

A transoral incisional biopsy was performed under general anesthesia, which resulted in mononucleated cells with ample cytoplasm, with scarce pleomorphism and few multinucleated giant cells, numerous foamy histiocytes and hemosiderin deposits, with positive immunohistochemistry (IHC) for CD-68, compatible with tenosynovial giant cell tumor.

Based on the foregoing, a combined surgical procedure was decided, via lateral cervicotomy and transoral approach and a possible Trotter-type access was considered if necessary. The procedure began with the cervicotomy, continuing medially to the carotid sheath and lateral to the pharynx, until exposing the retropharyngeal space and visualization of the upper edge of the tumor. The ipsilateral hypoglossal and superior laryngeal nerves were identified and preserved.

The bulging prevertebral muscles were identified and dissected to the tumor plane, without compromising the internal carotid or the vertebral arteries. En bloc resection of the tumor was achieved with an intraoperative biopsy that reports fusocellular tumor. Intraoperative endoscopy safety review revealed a disruption of the posterior wall of the nasopharynx, which was repaired with 3-0 vicryl. Nasogastric tube (NGS) was installed for feeding.

The patient evolved with mild dysphagia. Enteral feeding by NGS was started on the first postoperative day, well tolerated, progressing to oral feeding on the fourth postoperative day. Evaluation by the speech therapy team ruled out swallowing disorders, being discharged on the seventh day.

Biopsy reports a tumor measuring $5 \times 2 \times 1 \mathrm{~cm}$, partially encapsulated, composed of different types of cells, among which are multinucleated giant cells of the osteoclast type, interspersed with foamy histiocytes, siderophages and other smaller, homogeneous, ovoid histiocytic short cells, without atypia or mitosis, with little eosinophilic cytoplasm poorly defined. CD68 positive immunohistochemical staining in histiocytic cells and multinucleated giant cells and CD1A negative; compatible with Giant cell tumor of the tenosynovial type.

The patient remained asymptomatic on last check-up 6 months after surgery.
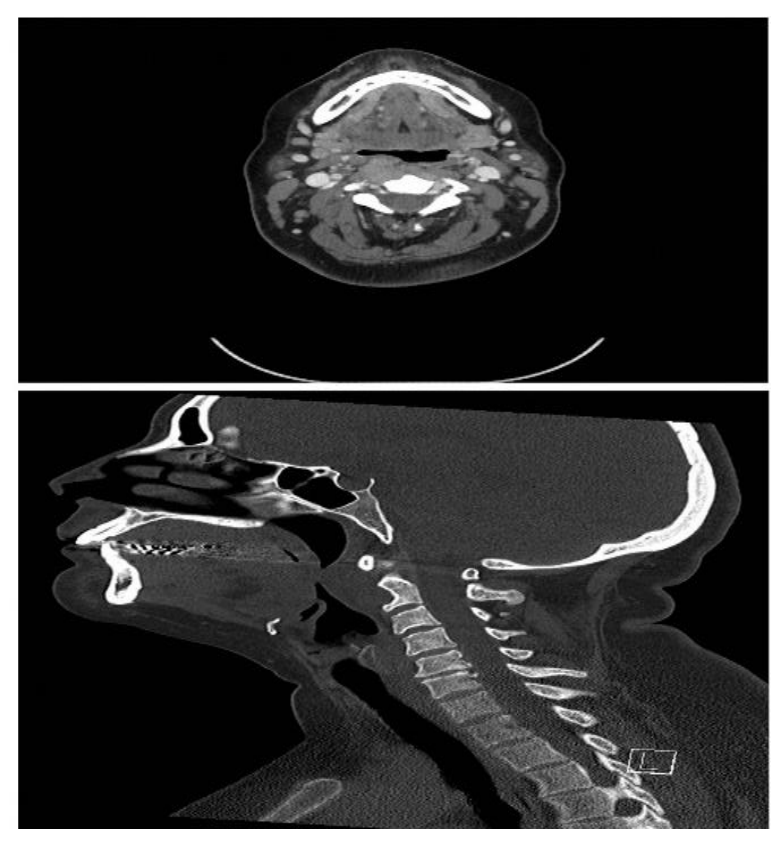

Figure 1: CT in axial and parasagittal plane, shows a lobulated mass located in the PVS at the height of C2. $98 \times 180 \mathrm{~mm}(300 \times 300 \mathrm{DPI})$.
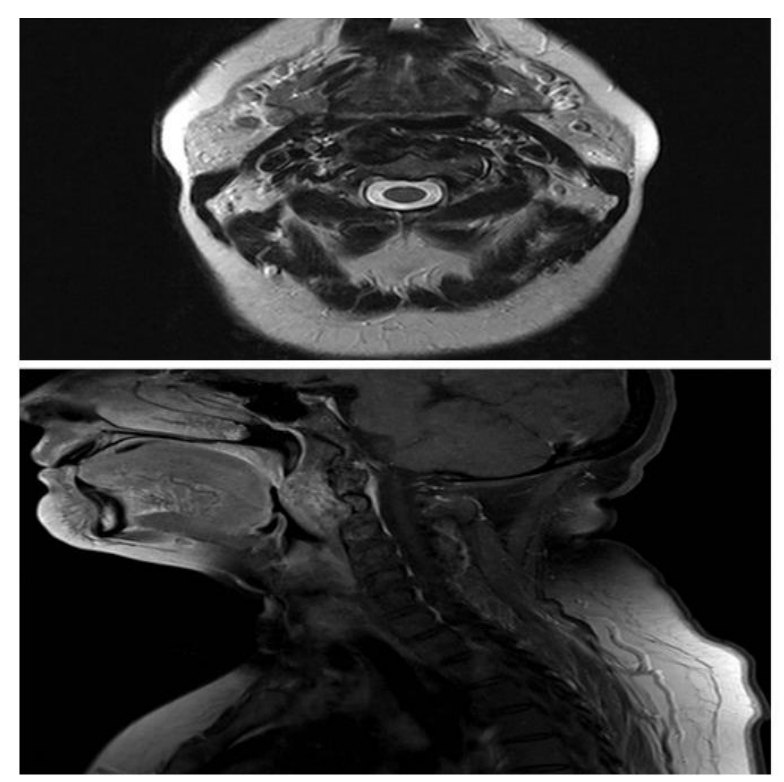

Figure 2: MR in axial and parasagittal plane, shows a heterogeneous and hypointense signal in T2-weighted sequences, suggesting a fibrous component and confirming the nonaggressive appearance of the mass. $98 \times 180 \mathrm{~mm}(300 \times 300 \mathrm{DPI})$.

\section{DISCUSSION}

The masses of the PVS and RPS have multiple etiologies, within these, primary tumors are possible, being malignant neoplasms extremely rare. ${ }^{1}$

Clinically, unlike tumors of the parapharyngeal space that generally grow asymptomatic, the anatomy of the RPS and PVS produces early symptoms due to mass effect. Thus, 
patients typically present with dysphagia, dyspnea or dysphonia. ${ }^{2}$ Since physical examination is limited, imaging studies play a fundamental role. At present, plain radiography and fluoroscopy have been replaced by CT and MR, techniques that allows locating the origin and extent. ${ }^{3}$ Oral sonographic studies can be used to guide fine needle biopsy or CT biopsy guidance for subzygomatic, premaxillary and retromandibular approaches.

Knowledge of cervical anatomy is of utmost importance. As our case shows, the close relationship of the mass and the longus colli muscle, as well as the displacement of adipose tissue allowed the tumor to be correctly located in the PVS. This, added to its particular imaging characteristics, limited the differential diagnoses to fibrous tumors, including the tenosynovial giant cell tumor.

The routine use of the cervical compartments, together with narrowing down the differential diagnoses, makes it possible to evaluate routes of spread, adjacent vital structures and the extent of the different pathological processes of the neck.

As previously mentioned, primary tumors of the RPS are extremely rare, mainly benign, with lipoma being the most common histological variety. In contrast to the above, the occupation of the RPS is more frequently due to the extension of neoplasms from naso and oropharynx. ${ }^{3}$ The possibility of nodal metastasis, secondary to the aforementioned carcinomas, as well as thyroid cancer or even melanoma, should not be ignored. ${ }^{4}$ There are also congenital lesions such as branchial cysts or ectopic parathyroids, vascular malformations, abscesses among others. Regarding the PVS, the presence of primary tumors in this region is equally rare, generally sarcomas of the longus colli/capitis muscles. ${ }^{3}$ As in the RPS, the PVS can also be invaded by carcinomas, mainly from the nasopharynx, oropharynx and hypopharynx. ${ }^{5}$ The existence of nerve sheath tumors, vascular malformations, abscesses, among others are also described. ${ }^{3}$

From the above, we can understand that the clinical/imaging differentiation of a tumor originating in PVS or RPS is of great difficulty despite the extensive anatomical knowledge, given the great proximity between both areas, and invasion produced by malignant tumors. For this reason, in addition to the mentioned studies, we must elucidate the origin of the tumor by determining the histology and intraoperative analysis.

Regarding the histological finding of the case presented, the giant cell tumor of the tenosynovial type (TTCG) constitutes a benign cell proliferation of the synovium, bursa and tendons. They are usually divided according to location (intra and/or extraarticular) and according to growth pattern (localized or diffuse). It generally affects the extremities and is very rarely found on the head and neck. Histologically, it has a nodular or villous pattern and presents two cell types: a group of small or large histiocytoid mononuclear cells and another of multinucleated giant cells. Small histiocytoid cells correspond to the predominant cell type in the tumor. Foamy histiocytes, hemosiderin deposits, and associated inflammatory infiltrate are frequently identified. Regarding the immunohistochemical profile, positivity is described for CD68, CD45 and CD163 in histiocytoid cells, CD68 and TRAP in multinucleated giant cells and clusterin in large mononucleated cells. ${ }^{6,7}$ This is consistent with the histological and immunohistochemical findings of the biopsy, in such a way that we believe that the primary origin of the tumor is from the PVS and in this context we emphasize that the preoperative differentiation of the origin can sometimes be tremendously complex. Malignant neoplasms such as liposarcoma and synovial sarcoma are even more rare. ${ }^{8}$

There are a number of surgical approaches to access the skull base and the anterior region of the upper cervical spine. These procedures include transcervicaltranstemporal approach, retraction and dissection of the soft palate, mandibular osteotomy, medial glossotomy, among others. However, the combination of mandibular osteotomy and glossopalatine section is often required to remove tumors located at previously reported sites. Trotter described a technique with a transmandibular and transoral section (mandibulotomy and glossotomy), which divides the oral cavity and floor of the mouth into two portions, one right and one left, broadly exposing the oropharynx. This procedure is very useful for treating oropharyngeal, parapharyngeal and retropharyngeal tumors, although it is not free of complications and sequelae such as salivary fistulas, dysphagia and hypoglossal nerve injury.

With the development of transoral robotic surgery (TORS) technique, first described by Weinstein in 2006, has gained a lot of ground in recent years for the confrontation of this type of tumors. ${ }^{9}$ The most relevant advantages when compared to conventional surgery are the reduction of extensive surgical approaches with the consequent damage to healthy neighboring structures, less compromise of the airway, reducing the need for tracheostomies and less swallowing disorders, while maintaining good oncological control of this type of injuries. ${ }^{10}$ In this case, an evaluation by a robotic surgery team was requested, but given the size, bone remodeling and proximity to the left vertebral arteries as well as both internal carotids, it was deemed not possible.

Being a benign pathology, during surgical planning we decided to carry out the least invasive approach possible, beginning with the dissection of the parapharyngeal space, in order to reach the retropharynx through the cervical route. Initial dissection took place medial to the carotid sheath, avoiding the hypoglossal and superior laryngeal nerves, only sectioning the superior thyroid artery in order to displace the pharynx and gain a good exposure to the base of $\mathrm{C} 1$. We had used this type of approach previously, for the anterior access of spinal surgery, but never for this type of tumor. The most commonly used techniques in this context are those described by Cloward, Smith and 
Robinson, and Bayley and Badley, where the most frequently described complications are dysphagia secondary to traction of the esophagus and surgical edema. Rarer complications are esophageal or pharyngeal perforations, which often go unnoticed intraoperatively and manifest themselves in the postoperative course as fistulas or sepsis. Fortunately, the injury in our case was detected in the operative act and was repaired without much difficulty. Another form of complication in this type of approach is related to the injury of the superior and recurrent laryngeal nerves, the latter being the one that produces the largest form of legal complaints at present. Less frequent is the injury of the carotid artery, its rupture is very infrequent and can be devastating. It should be considered that intraoperative mobilization of this artery is associated to the detachment of atheroma plaques, which may lead to embolic infarcts in the brain. Vertebral artery injury is even rarer. ${ }^{11}$

In our case, this approach allowed adequate exposure, and permitted complete tumor in block resection, and without associated vascular injury. With this, we were able to avoid the access described by Trotter, which although provides excellent exposure (especially in cases of malignant tumors), is also a surgery with greater potential for morbidity. We believe that in order to optimize the postoperative evolution, it is essential that prior to the end of the surgery a feeding tube must be advanced, in order to restart early feeding, regardless of the postoperative evolution.

\section{CONCLUSION}

As it is a low-frequency pathology, the literature regarding these type of tumors in RPS and PVS is scarce and there are no meta-analyzes or randomized studies that compare the advantages of one surgical technique over the other. Treatment can vary, presenting multiple alternatives: from modulating therapies to radiotherapy and finally surgery. This last option seems to be the ideal treatment in the management of tumors with this histological variety, considering the lower morbidity and sequelae for the patient. Compared to the classic surgical approach of the skull base and anterior cervical spine, the described transcervical-transoral approach is, in our opinion, equally effective and less morbid, as an alternative in the treatment of these type of lesions.

\section{ACKNOWLEDGEMENTS}

Authors would like to acknowledge the Hospital Clínico Universidad de Chile's department of pathology and imagenology for their assistance, specially Dr. Marcelo López and Dr. José Manuel Herranz.
Funding: No funding sources

Conflict of interest: None declared

Ethical approval: Not required

\section{REFERENCES}

1. Gundelach R, Ullah R, Coman S, Campbell K. Liposarcoma of the retropharyngeal space. J Laryngol Otol. 2005;119(08):651-4.

2. Gallo S, Bandi F, Maffioli MP, Giudice M, Castelnuovo P, Fazio E, et al. Retropharyngeal space schwannoma: a rare entity. Iranian J Otorhinolaryngol. 2017;29(6):95.

3. Debnam J, Guha-Thakurta N. Retropharyngeal and prevertebral spaces: anatomic imaging and diagnosis. Otolaryngol Clin North Am. 2012;45(6):1293-310.

4. Davis WL, Harnsberger HR, Smoker WR, Watanabe AS. Retropharyngeal space: evaluation of normal anatomy and diseases with CT and MR imaging. Radiology. 1990;174(1):59-64.

5. Imre A, Pinar E, Erdogan N, Ata Ece A, Olgun Y, Aladag I, et al. Prevertebral Space Invasion in Head and Neck Cancer. Ann Otol Rhinol Laryngol. 2014;124(5):378-83.

6. Fletcher C, Bridge J, Hogendoorn P, Mertens F. WHO Classification of Tumours of Soft Tissue and Bone. World Health Organization. 2013;100-3.

7. Goldblum J, Folpe A, Weiss S. Benign Tumors and Tumor-like Lesions of Synovial Tissue. In: Enzinger \& Weiss's. Soft Tissue Tumors, 6th ed. Philadelphia, PA: Elsevier Saunders. 2014;766-77.

8. Somerhausen NS, Dal Cin P. Diffuse-type giant cell tumor: Clinicopathologic and immunohistochemical analysis of 50 cases with extraarticular disease. Am J Surg Pathol. 2000;24:479-92.

9. Genden EM, O'Malley BW, Weinstein GS, Stucken CL, Selber JC, Rinaldo A, et al. Transoral robotic surgery: role in the management of upper aerodigestive tract tumors. Head Neck. 2012;34:88693.

10. Nichols AC, Yoo J, Hammond JA, Fung K, Winquist E, Read N, et al. Earlystage squamous cell carcinoma of the oropharynx: radiotherapy vs transoral robotic surgery (ORATOR)-study protocol for a randomized phase II trial. BMC Cancer. 2013;13:13.

11. García-Armengol R, Colet-Esquerre S, TeixidorRodríguez P, Alamar-Abril M, Cladellas-Ponsa JM, Hostalot-Panisello C, et al. Complicaciones del abordaje anterior en la patología de la columna cervical. Neurocirugía. 2007;18:209-20.

Cite this article as: Rappoport DA, Fuenzalida L, Sepulveda PI, Gac PJ. Giant cell tumor of the tenosynovial type in the prevertebral space with a cervical approach. Int J Otorhinolaryngol Head Neck Surg 2022;8:81-4. 